\title{
Single-Atom Source in the Picokelvin Regime
}

\author{
A. G. Manning, R. Khakimov, R. G. Dall, and A. G. Truscott \\ Research School of Physics and Engineering, Australian National University, \\ Canberra, Australian Capital Territory 0200, Australia
}

(Received 16 April 2014; revised manuscript received 4 July 2014; published 24 September 2014)

\begin{abstract}
An important aspect of the rapidly growing field of quantum atom optics is exploring the behavior of ultracold atoms at a deeper level than the mean field approximation, where the quantum properties of individual atoms becomes important. Major recent advances have been achieved with the creation and detection of reliable single-atom sources, which is a crucial tool for testing fundamental quantum processes. Here, we create a source comprised of a single ultracold metastable helium atom, which enables novel free-space quantum atom optics experiments to be performed with single massive particles with large de Broglie wavelengths.
\end{abstract}

DOI: 10.1103/PhysRevLett.113.130403

PACS numbers: 03.75.Be, 34.50.Fa, 37.10.De, 42.50.Dv

The rapid development of ultracold-atom physics since the invention of the optical laser has facilitated many experiments that probe fundamental quantum phenomena. Several of the most striking results require the ability to measure individual atoms, where quantum-statistical properties such as the Hanbury Brown-Twiss effect [1,2], Hong-Ou-Mandel interferometry [3], as well as manyparticle interference [4] can be observed. Another key aspect to testing quantum mechanics is the creation of controllable single-particle sources. From G. I. Taylor's landmark demonstration that Young's double-slit experiment still displays a diffraction pattern if there is on average less than one photon present [5] (which was verified a long time later with a truly single-photon source [6]), to tests of Bell's inequalities [7,8], experiments measuring individual photons have played a pivotal role in testing the predictions of quantum mechanics.

The first trapped single-atom sources were typically loaded directly from a magneto-optical trap, by methods such as using an optical dipole trap with an extremely small focused spot size to catch single ${ }^{87} \mathrm{Rb}$ atoms [9] or loading Cs atoms into a standing wave dipole trap with the ability to adiabatically transport atoms [10]. Other experiments have loaded single atoms into an array of separate sites [11-13] where atoms can be imaged in situ [13], and experiments have shown novel phenomena such as the superfluid to Mott insulator transition [14] at the single-atom level [15]. Single atoms have also been confined near surfaces to form hybrid quantum devices [16]. A further improvement to these single-atom sources is the use of precisely controlled light-assisted collisions, which can increase the loading efficiency of single atoms from the typical rate limited by pairwise loss of $50 \%$ to $91 \%$ [17] for ${ }^{85} \mathrm{Rb}$. Recently, there has been an effort to reduce the energy spread of these sources, using Raman sideband cooling to cool single ${ }^{87} \mathrm{Rb}$ atoms close to the three-dimensional ground state of an optical tweezer $[18,19]$, so as to make quantum atom optic experiments possible.
In this Letter, we present a method by which an ultracold single-atom bosonic source can be created by significantly increasing the two-body losses within in a Bose-Einstein condensate (BEC). The resulting single metastable helium $\left(\mathrm{He}^{*}\right)$ atom source has a three-dimensional ground-state occupation of $\sim 50 \%$ and a temperature of $\sim 890 \mathrm{pK}$ as measured by its averaged time-of-flight (TOF) profile. While our system is comprised of $\mathrm{He}^{*}$, our method should be readily adaptable to other optical BEC experiments, in which a large two-body loss rate can be instigated. Furthermore, because of the lack of nuclear spin of $\mathrm{He}^{*}$, our source that is in the state $\left|J, m_{J}\right\rangle=|1,0\rangle$ is essentially nonmagnetic, making it an interesting candidate for hybrid quantum systems [16]. Finally, the source demonstrated here is ideally suited to realizations of fundamental tests of quantum mechanics with freely propagating massive particles, where large de Broglie wavelengths as well as singleatom detection with high temporal resolution [20] are required. Such tests include investigations of Wheeler's delayed-choice experiment, which was recently demonstrated with photons [21], or an atomic version of entanglement, as was originally envisioned in the famous paper of Einstein, Podolsky, and Rosen (EPR) [22].

To create our source of single $\mathrm{He}^{*}$ atoms, we first precool the atoms in a magnetic trap, which can only retain atoms in the $|1,1\rangle$ state and, thus, automatically spin polarizes the gas, resulting in the suppression of Penning ionization [20]. However, by loading the cloud into an optical dipole trap, atoms of any spin state can be held. After spin flipping atoms held in the dipole trap with the application of rf radiation from the $|1,1\rangle$ state to the $|1,0\rangle$ state, the number of trapped atoms rapidly decays due to the extremely short lifetime of the unpolarized metastable helium atoms. This unpolarized lifetime is orders of magnitude shorter than all other lifetimes in the system, including the single-body lifetime limited by background collisions and the radiative lifetime of the metastable helium excited state (7870 s [23]). After holding 
the cloud in this trap for a time long compared to the time scale over which two-body losses deplete the trap population, a single atom is expected in 50\% of experimental cycles, and zero atoms otherwise, depending on whether an odd or even number of atoms was in the trap initially.

The change in population $N$ of a cloud of $\mathrm{He}^{*}$ atoms loaded at a rate $L$ can be characterized by

$$
\frac{d N(t)}{d t}=L-\alpha N(t)-\beta \int \rho^{2}(\mathbf{r}, t) d^{3} \mathbf{r} .
$$

The single-body loss rate $\alpha$ depends on collisions with background particles, while the two-body loss rate $\beta$ is dominated by Penning ionization. The density of ultracold trapped clouds is typically insufficient for collisions involving three or more particles to contribute to this loss rate. The population of the cloud as a function of time can be determined by removing the loading source and integrating over the cloud characterized by an effective volume $V$, such that [24]

$$
N(t)=\frac{2^{3 / 2} \alpha N(0) V}{\left[2^{3 / 2} \alpha V+\beta N(0)\right] \exp (\alpha t)-\beta N(0)} .
$$

Recent experimental measurements have found that $\beta\left(m_{J}=1, m_{J}^{\prime}=1\right)=(2.9 \pm 2.0) \times 10^{-14} \mathrm{~cm}^{3} \mathrm{~s}^{-1}$ for pairs of atoms with spins $m_{J}$ and $m_{J}^{\prime}$ [25]. In contrast, the unpolarized loss rates have been measured to be $\beta(0,0)=$ $(6.6 \pm 0.4) \times 10^{-10} \mathrm{~cm}^{3} \mathrm{~s}^{-1}$ for pairs of atoms in the $\left|J=1, m_{J}=0\right\rangle \quad$ state, and $\beta(-1,1)=(7.4 \pm 1.0) \times$ $10^{-10} \mathrm{~cm}^{3} \mathrm{~s}^{-1}$ for collisions between $\left|1, m_{J}=-1\right\rangle$ and $\left|1, m_{J}^{\prime}=1\right\rangle$ atoms [26]. The reduction in the Penning ionization loss rate of 4 orders of magnitude for spin polarized as compared to unpolarized metastable helium atoms has enabled a BEC to be reached [27-30].

The experimental setup is largely based on that in Ref. [31] and is depicted in Fig. 1. Before loading atoms into the optical trap, ultracold ${ }^{4} \mathrm{He}^{*}$ in the $|1,1\rangle$ state is magnetically trapped and evaporatively cooled [30] to just below the BEC transition temperature $(\sim 1 \mu \mathrm{K})$. Around $10^{4}$ atoms are then transferred into a vertical optical dipole trap by ramping up over $200 \mathrm{~ms}$ the intensity of a far-red detuned $(1550 \mathrm{~nm})$ focused laser beam in the direction of gravity. The magnetic trap is switched off, and the only magnetic field present is generated by our magnetic field stabilization "nullerometer" [32], which provides a bias magnetic field $\sim 1 \mathrm{G}$. Then, the vertical dipole trap is ramped down over $100 \mathrm{~ms}$, resulting in a trap with harmonic frequencies of $\left(\omega_{x}, \omega_{y}, \omega_{z}\right) \approx 2 \pi \times(1800,1800,12) \mathrm{Hz}$. The trap depth (equivalent to a temperature of a few nanokelvin) is then held at this point for $2 \mathrm{~s}$, which allows the majority of the thermal atoms to exit the trap.

A $\sim 35 \mu$ s radio-frequency pulse is then used to transfer a portion of the $|1,1\rangle$ atoms to the $|1,0\rangle$ state. To remove any remaining population in the $|1, \pm 1\rangle$ state, a large magnetic field gradient is applied. After holding the atoms in the trap
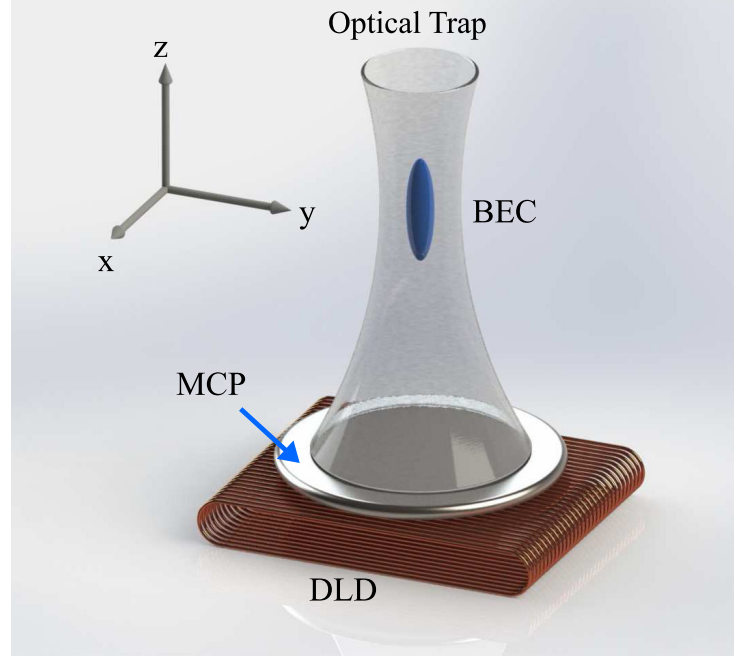

FIG. 1 (color online). Atoms held in the dipole trap after transfer from the magnetic trap can be released from the trap to fall under gravity to the microchannel plate (MCP) and delay-line detector (DLD) situated below.

to allow two-body losses to deplete the cloud, we release the atoms to fall under gravity to the MCP and DLD located $\sim 850 \mathrm{~mm}$ below by switching off the optical trapping beam.

A measurement of the trap lifetime is shown in Fig. 2, where the atoms were held in the trap for different durations between the spin flipping and trap switch off. The bimodal nature of the trap loss is evident from the two distinctly

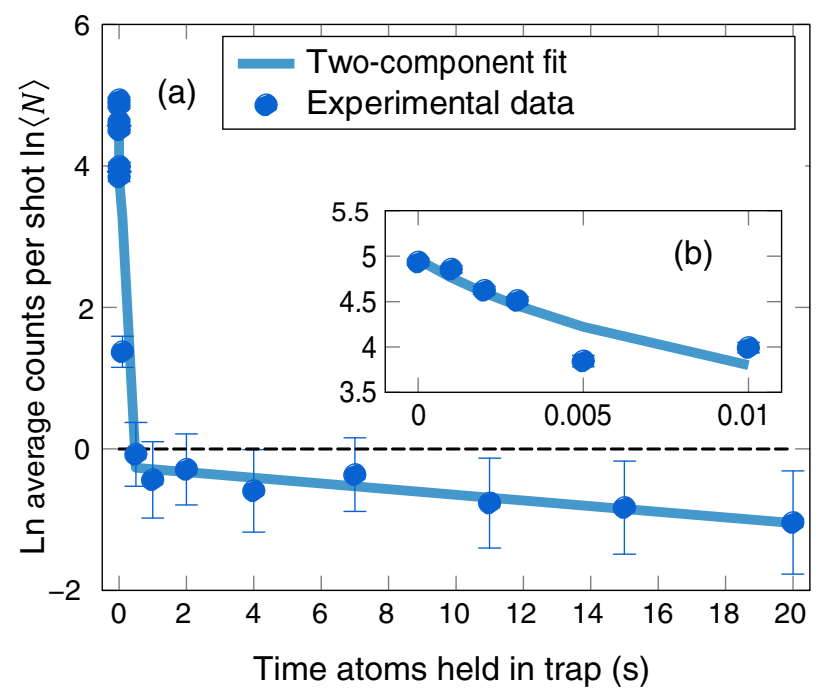

FIG. 2 (color online). Logarithm of the trap population, adjusted by a quantum efficiency (QE) of detection of $\sim 20 \%$, of $m_{F}=0$ atoms as a function of time held in the trap. For times of less than $0.5 \mathrm{~s}$ [inset (b)], the two-body loss rate is dominant, while for later times (a) the single-body loss rate becomes dominant and fits to a $1 / e$ lifetime of $24.9 \mathrm{~s}$. The error bars indicate statistical uncertainty, and the dashed line shows the threshold below which no more than a single atom is present in the trap on average. 
different loss rates occurring before and after hold times of $\sim 0.5 \mathrm{~s}$; initially, there is rapid loss due to binary collisions, which subsides at later times as the population of the trapped cloud is reduced such that one-body losses dominate.

The one- and two-body loss rates ( $\alpha$ and $\beta$ ) for the cloud can be derived from the bimodal fit to the trap population as a function of time [Eq. (2)] illustrated in Fig. 2. In this case, the two-body loss rate $\beta$ is set to zero once the average count rate drops below one atom per iteration (adjusted for detection efficiency) after a hold time of $0.5 \mathrm{~s}$. The biggest uncertainty in determining $\beta$ is from accurately characterizing the trap volume $V$, which is assumed to take on an ideal Bose gas density profile and has an error of $20 \%$. We find from our fit that $\alpha=0.040 \pm 0.008$, which corresponds to a one-body lifetime of $1 / \alpha=24.9 \mathrm{~s}$, and a value for the twobody loss rate of $\beta(0,0)=4.9 \pm 1.3 \times 10^{-10} \mathrm{~cm}^{3} / \mathrm{s}$, which is in reasonable agreement with the result obtained from previous measurements [26] taking into account the absence of Hanbury Brown-Twiss "bunching" in a BEC and the assumptions used.

To determine whether a true single-atom source is being produced, we repeat the experiment for over 8000 experimental cycles. Adjusting for a quantum efficiency for our detector of $\sim 20 \%$, which we have measured independently using a relative number squeezing process (similar to that in Ref. [33]), we effectively observe on average a single count on our detector for every two runs of the experiment. With knowledge of the time and spatial position with which the atom(s) should land on the detector, we integrate the counts over a small $(\Delta t, \Delta x, \Delta y)=(2 \mathrm{~ms}, 20 \mathrm{~mm}, 20 \mathrm{~mm})$ volume to reduce the effect of dark counts. Within this volume, over the 8000 experimental cycles we expect $2 \%$ of the observed counts to be due to dark counts.

To characterize the fidelity of our single-atom source, we calculate the normalized second-order correlation function: the probability of detecting two atoms as a function of experimental iterations, normalized by the average atom intensity squared. A measurement of this is shown in Fig. 3 and tests if any pair of particles measured is more likely to occur by one particle being in each of two separate experimental iterations, rather than both the particles occurring in the same iteration. We measure $g^{(2)}(0)=0$ for our single-atom source, which demonstrates that we have a strongly sub-Poissonian source.

To determine the temperature of our source, we fit Gaussians to the TOF distributions accumulated over many runs of the experiment (see Fig. 4). In the transverse direction $(x)$ the width of the Gaussian distribution we measure is consistent with that expected for a ground-state distribution. In the longitudinal $(z)$ direction, the observed TOF profile fits to a temperature of $890 \mathrm{pK}$, where to calculate the temperature we have used the fitted width of the Gaussian distribution and subtracted off the zero-point energy. To determine the ground-state occupation in this

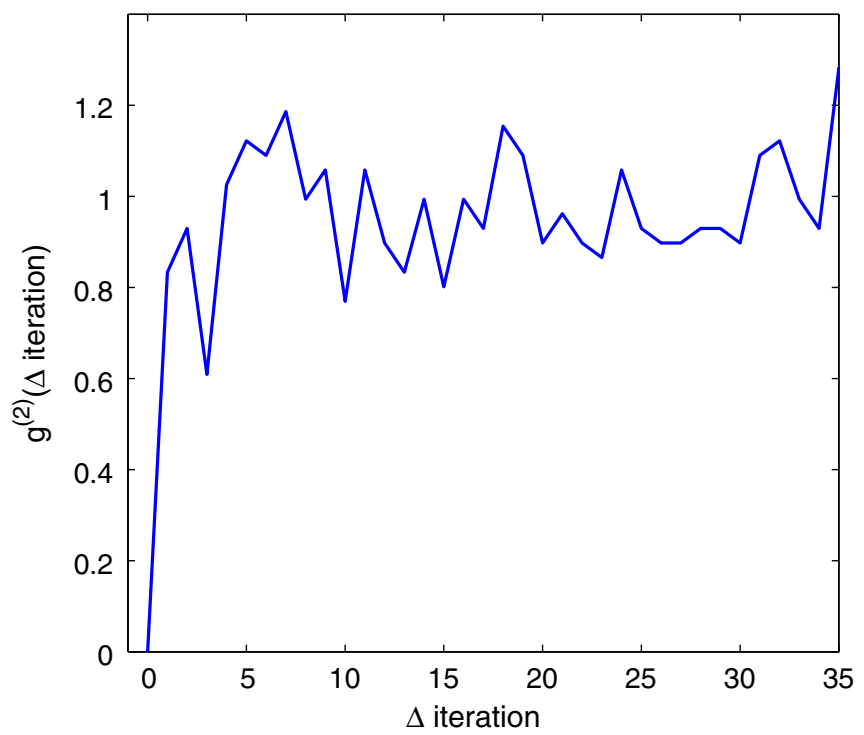

FIG. 3 (color online). Second-order correlation function $g^{(2)}$ measured for the single-atom source as a function of increment between experimental iterations.

direction, we perform an optimized fit using the first five states of the harmonic oscillator while constraining the fit parameter to a trapping frequency of $12 \mathrm{~Hz}$, which was measured independently. From this fit, we determine that there is $50 \%$ occupancy in the longitudinal ground state.

In summary, we present a single $\mathrm{He}^{*}$ atom source in the picokelvin energy regime that should be readily adaptable to other optical BEC experiments. At present, the source has some limitations, i.e., long duty cycle ( $\sim 30 \mathrm{~s}), 20 \%$ detector efficiency, compared to previously reported singleatom sources. However, both of these are not fundamental constraints, since the duty cycle can be made much shorter by improving the evaporation efficiency and the $\mathrm{QE}$ for $\mathrm{He}^{*}$ detection can, in principle, approach unity in some applications [34]. Despite these limitations, our source is ideally suited to realizations of fundamental tests of quantum
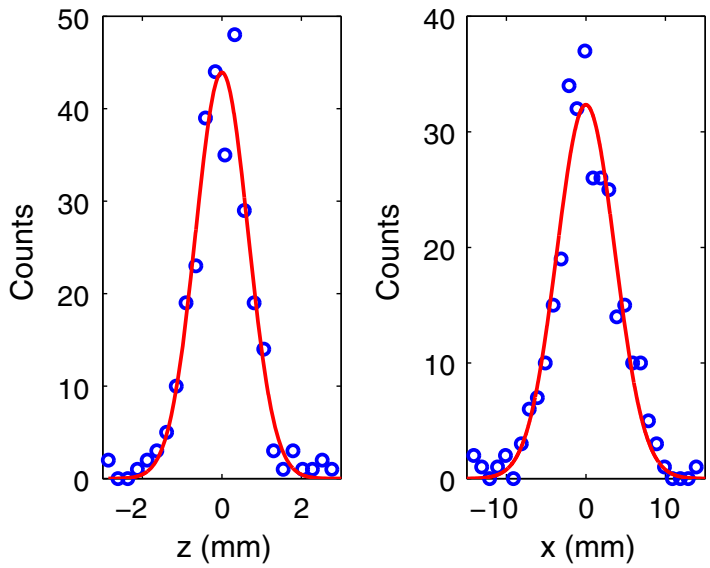

FIG. 4 (color online). Integrated momentum distributions measured after time of flight, with associated Gaussian fits. 
mechanics with freely expanding massive particles. For example, it should be possible to extend our method to produce a double-well optical trap, thus, creating a pair of single atoms. If the double well is created along the optical beam axis, then one atom can be guided [35] into the other and an entangled pair created via a collision [36]. The collision probability is proportional to the square of the density of the colliding particles, which for the case of single-atom sources is small. However, one might be able to use a low field Feshbach resonance for $\mathrm{He}^{*}$ atoms [37] to increase the scattering length by a factor of $\sim 50$ and, consequently, the collision probability to unity. Such an experiment would generate a freely propagating entangled state of massive particles, in which their external degrees of freedom are entangled, as was originally proposed in the EPR paper [22].

A. G. T acknowledges the support of the Australian Research Council through the Future Fellowship Grant No. FT100100468.

[1] M. Schellekens, R. Hoppeler, A. Perrin, J. Viana Gomes, D. Boiron, A. Aspect, and C. I. Westbrook, Science 310, 648 (2005).

[2] S. S. Hodgman, R. G. Dall, A. G. Manning, K. G. H. Baldwin, and A. G. Truscott, Science 331, 1046 (2011).

[3] A. M. Kaufman, B. J. Lester, C. M. Reynolds, M. L. Wall, M. Foss-Feig, K. R. A. Hazzard, A. M. Rey, and C. A. Regal, Science 345, 306 (2014).

[4] R. G. Dall, A. G. Manning, S. S. Hodgman, W. RuGway, K. V. Kheruntsyan, and A. G. Truscott, Nat. Phys. 9, 341 (2013).

[5] G. I. Taylor, Proc. Cambridge Philos. Soc. 15, 114 (1909).

[6] P. Grangier, G. Roger, and A. Aspect, Europhys. Lett. 1, 173 (1986).

[7] J. F. Clauser and A. Shimony, Rep. Prog. Phys. 41, 1881 (1978).

[8] A. Aspect, P. Grangier, and G. Roger, Phys. Rev. Lett. 47, 460 (1981).

[9] N. Schlosser, G. Reymond, I. Protsenko, and P. Grangier, Nature (London) 411, 1024 (2001).

[10] S. Kuhr, W. Alt, D. Schrader, M. Müller, V. Gomer, and D. Meschede, Science 293, 278 (2001).

[11] R. Dumke, M. Volk, T. Müther, F. B. J. Buchkremer, G. Birkl, and W. Ertmer, Phys. Rev. Lett. 89, 097903 (2002).

[12] D. Schrader, I. Dotsenko, M. Khudaverdyan, Y. Miroshnychenko, A. Rauschenbeutel, and D. Meschede, Phys. Rev. Lett. 93, 150501 (2004).

[13] K. D. Nelson, X. Li, and D. S. Weiss, Nat. Phys. 3, 556 (2007).

[14] M. Greiner, O. Mandel, T. Esslinger, T. Hänsch, and I. Bloch, Nature (London) 415, 39 (2002).

[15] W. S. Bakr, A. Peng, M. E. Tai, R. Ma, J. Simon, J. I. Gillen, S. Fölling, L. Pollet, and M. Greiner, Science 329, 547 (2010).
[16] J. D. Thompson, T. G. Tiecke, N. P. de Leon, J. Feist, A. V. Akimov, M. Gullans, A. S. Zibrov, V. Vuletic, and M. D. Lukin, Science 340, 1202 (2013).

[17] A. V. Carpentier, Y. H. Fung, P. Sompet, A. J. Hilliard, T. G. Walker, and M. F. Andersen, Laser Phys. Lett. 10, 125501 (2013).

[18] A. M. Kaufman, B. J. Lester, and C. A. Regal, Phys. Rev. X 2, 041014 (2012).

[19] J. D. Thompson, T. G. Tiecke, A. S. Zibrov, V. Vuletic, and M. D. Lukin, Phys. Rev. Lett. 110, 133001 (2013).

[20] W. Vassen, C. Cohen-Tannoudji, M. Leduc, D. Boiron, C. I. Westbrook, A. Truscott, K. Baldwin, G. Birkl, P. Cancio, and M. Trippenbach, Rev. Mod. Phys. 84, 175 (2012).

[21] V. Jacques, E. Wu, F. Grosshans, F. Treussart, P. Grangier, A. Aspect, and J.-F. Roch, Science 315, 966 (2007).

[22] A. Einstein, B. Podolsky, and N. Rosen, Phys. Rev. 47, 777 (1935).

[23] S. S. Hodgman, R. G. Dall, L. J. Byron, K. G. H. Baldwin, S. J. Buckman, and A. G. Truscott, Phys. Rev. Lett. 103, 053002 (2009).

[24] A. Browaeys, J. Poupard, A. Robert, S. Nowak, W. Rooijakkers, E. Arimondo, L. Marcassa, D. Boiron, C. I. Westbrook, and A. Aspect, Eur. Phys. J. D 8, 199 (2000).

[25] O. Sirjean, S. Seidelin, J. V. Gomes, D. Boiron, C. I. Westbrook, A. Aspect, and G. V. Shlyapnikov, Phys. Rev. Lett. 89, 220406 (2002).

[26] G. B. Partridge, J.-C. Jaskula, M. Bonneau, D. Boiron, and C. I. Westbrook, Phys. Rev. A 81, 053631 (2010).

[27] A. Robert, O. Sirjean, A. Browaeys, J. Poupard, S. Nowak, D. Boiron, C. I. Westbrook, and A. Aspect, Science 292, 461 (2001).

[28] F. Pereira Dos Santos, J. Léonard, J. Wang, C. J. Barrelet, F. Perales, E. Rasel, C. S. Unnikrishnan, M. Leduc, and C. Cohen-Tannoudji, Phys. Rev. Lett. 86, 3459 (2001).

[29] A. S. Tychkov, T. Jeltes, J. M. McNamara, P. J. J. Tol, N. Herschbach, W. Hogervorst, and W. Vassen, Phys. Rev. A 73, 031603(R) (2006).

[30] R. G. Dall and A. G. Truscott, Opt. Commun. 270, 255 (2007).

[31] R. G. Dall, S. S. Hodgman, A. G. Manning, M. T. Johnsson, K. G. H. Baldwin, and A. G. Truscott, Nat. Commun. 2, 291 (2011).

[32] C. J. Dedman, R. G. Dall, L. J. Byron, and A. G. Truscott, Rev. Sci. Instrum. 78, 024703 (2007).

[33] J.-C. Jaskula, M. Bonneau, G. B. Partridge, V. Krachmalnicoff, P. Deuar, K. V. Kheruntsyan, A. Aspect, D. Boiron, and C. I. Westbrook, Phys. Rev. Lett. 105, 190402 (2010).

[34] F. B. Dunning, A. C. H. Smith, and R. F. Stebbings, J. Phys. B 4, 1683 (1971).

[35] R. G. Dall, S. S. Hodgman, M. T. Johnsson, K. G. H. Baldwin, and A. G. Truscott, Phys. Rev. A 81, 011602(R) (2010).

[36] A. Perrin, H. Chang, V. Krachmalnicoff, M. Schellekens, D. Boiron, A. Aspect, and C. I. Westbrook, Phys. Rev. Lett. 99, 150405 (2007).

[37] M. R. Goosen, T. G. Tiecke, W. Vassen, and S. J. J. M. F. Kokkelmans, Phys. Rev. A 82, 042713 (2010). 